\title{
Tackling periodontitis in patients
}

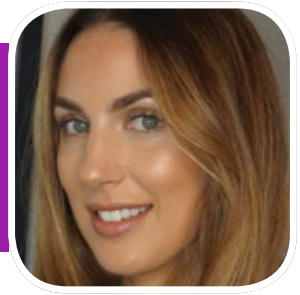

\author{
Claire Berry, dental hygienist and Ambassador for the British \\ Society of Dental Hygiene and Therapy (BSDHT), explains how \\ she treats patients with periodontitis, and why more professionals \\ should be exploring different approaches to gum disease in order \\ to get the best results.
}

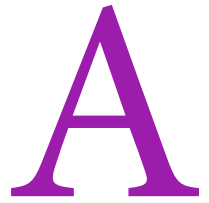

lthough it's difficult to assess how many individuals currently have periodontitis in the UK, it's generally assumed that at least $45 \%$ of individuals have some form of gum disease. ${ }^{1}$ Of course, a large amount of this is likely to be gingivitis - but as this is the precursor to periodontitis, is it any less important?

\section{Prevention first}

We all know that periodontitis is preceded by gingivitis, and yet I still think that some clinicians aren't placing enough importance on prevention and the main thing that causes the issues - the biofilm. It's common knowledge that once gingivitis has progressed into periodontitis the damage cannot be undone, so treatment from this point really needs to be about stabilising the condition so that it doesn't worsen and cause more suffering and expense for the patient.

Even a Basic Periodontal Examination (BPE) score of 1 should ring alarm bells what we need to realise is that any bleeding, no matter how small, is evidence of an immune response in the patient. The immune response to bacteria is eventually what leads to bone loss and other irreversible changes, so we need to be able to stop this as soon as possible.

\section{Treatment for periodontitis}

I tend to take a unique approach to treating periodontitis and flip the usual appointment order around to facilitate better results. Basically, using methods I have learned from experienced and skilled clinicians, the first step after recording BPE and carrying out bleeding scores is to introduce Oral Hygiene Instruction $(\mathrm{OHI})$ there and then with the help of disclosing the biofilm.
This way, patients can easily visualise the current state of their mouth and see how the biofilm is leading to periodontitis and further problems in the oral cavity. I use disclosing solution as this perfectly highlights the biofilm and reveals all of the bacteria to patients. I often find this step gives them a jolt of realisation as it's so visual - it's easy to ignore what you can't see, but once it's visible the problem becomes more real.

I then teach people how to remove this biofilm properly, so that they can perform excellent oral care at home twice a day. It allows me to tailor my advice to suit their current situation. I also remove all of the biofilm currently present both above and below the gingiva and tell them that this will begin to form again not long after I have removed it- this way I know we're starting on a clean slate but demonstrates that they must continue with removing it daily and it encourages them to take an active part in stabilising their own periodontitis.

I find this method really helps in the long run, as when patients return (six weeks later) there should be considerably less inflammation, swelling, bleeding etc. This means that I can take more accurate readings and assess the extent of the problem better as there will be no other factors impacting the readings. Following this, I ask patients to return every three months, routinely highlighting or disclosing the biofilm and ensuring that they are following the advice given to stabilise the condition properly.

Stable and unstable periodontitis will need different treatment approaches. If a patient is already stable, routine check-ups are necessary to ensure that they remain so, and that their condition doesn't deteriorate. On the other hand, those suffering from unstable periodontitis absolutely must have their conditions brought under control.
The role of dental hygienists and dental therapists

Dental hygienists and dental therapists are the best placed people in the practice to stabilise and prevent periodontitis. Despite this, I think we are still being overlooked in our profession by dentists and not enough awareness exists among patients as to what our roles actually involve.

I've long believed that by implementing better skill mix and by championing the skills of dental hygienists and dental therapists to their full extent, practices will see better results for patients, less litigation and a more streamlined approach to care. We cannot properly help patients with periodontitis if we don't have the correct amount of time and resources available, so this is something that needs to be understood in order for things to improve.

I was excited to be chosen as a BSDHT Ambassador because it's going to give me a chance to really show my passion for the profession and provide a good platform to speak about issues such as this. Many voices shout louder than a single voice alone, so I think it's really important for people in the profession to join Societies which allow them to have this representation. The BSDHT is exceptional as it is always fighting our corner and always covers things which are of the utmost importance for us all.

For more information about the BSDHT, visit www.bsdht.org.uk, call 01788575050 or email enquiries@bsdht.org.uk.

\section{Reference}

1. British Society of Periodontology. Gum Health Resources for Patients. Available at: https://www.bsperio.org.uk/patients/ (accessed January 2020).

https://doi.org/10.1038/s41407-020-0314-0 